\title{
LHC data and its impact on nCTEQ15 PDFs
}

\section{The nCTEQ Collaboration:* $\quad$ D. B. Clark, E. Godat, T. Ježo, C. Keppel,}

K. Kovařík, A. Kusina, ${ }^{5,6}$ F. Lyonnet, J.G. Morfin, F. I. Olness, ${ }^{1 \dagger}{ }^{1}$ J.F. Owens, I. Schienbein, J. Y. Yu ${ }^{1}$

${ }^{1}$ Southern Methodist University, Dallas, TX 75275, USA

${ }^{2}$ Physik-Institut, Universität Zürich, Winterthurerstrasse 190, CH-8057 Zürich, Switzerland

${ }^{3}$ Thomas Jefferson National Accelerator Facility, Newport News, VA, 23606, USA

${ }^{4}$ Institut für Theoretische Physik, Westfälische Wilhelms-Universität Münster,

Wilhelm-Klemm-Straße 9, D-48149 Münster, Germany

${ }^{5}$ Laboratoire de Physique Subatomique et de Cosmologie, Université Grenoble-Alpes,

CNRS/IN2P3, 53 avenue des Martyrs, 38026 Grenoble, France

6Institute of Nuclear Physics, Polish Academy of Sciences,

ul. Radzikowskiego 152, 31-342 Cracow, Poland

${ }^{7}$ Fermi National Accelerator Laboratory, Batavia, Illinois 60510, USA

${ }^{8}$ Department of Physics, Florida State University, Tallahassee, Florida 32306-4350, USA

The LHC heavy ion data for $W / Z$ production can provide new incisive information on the PDFs. This data is sensitive to the heavier quark flavors (strange and charm) in a high energy kinematic region; this can facilitate the determination of PDFs in the small $x$ region where previous data was limited. At present, the flavor separation of the proton PDFs is dependent on DIS data from nuclear targets. Therefore, improved nuclear corrections can also yield enhanced flavor determination of both the proton and nuclear PDFs.

XXV International Workshop on Deep-Inelastic Scattering and Related Subjects

3-7 April 2017

University of Birmingham, $U K$

${ }^{*}$ We acknowledge the hospitality of CERN, DESY, and Fermilab where a portion of this work was performed. This work was also partially supported by the U.S. Department of Energy under Grant No. DE-SC0010129.

${ }^{\dagger}$ Speaker. 


\section{Introduction}

In May 2015, the LHC reached a record setting energy of $13 \mathrm{TeV}$ for proton-proton collisions; it is likely the LHC will continue to hold the energy record for the foreseeable future until it is surpassed by a FCC facility. Therefore, our search for "new physics" phenomena turns to a careful analysis of the accumulating LHC data to make incisive comparisons between experimental measurements and theoretical predictions. The Parton Distribution Functions (PDFs) are the key ingredient that enables us to connect experiment with theory by applying the QCD improved parton model to describe the distribution of quarks and gluons in the proton.

Despite decades of studies, there is yet much to learn about the proton structure. A very interesting new result which was presented at this meeting was the ratio of the strange PDF to the up- and down-sea quark PDFs, as shown in Fig. 1. As the strange quark is heavier than the up and down quarks, the common expectation was that the strange PDF would be suppressed relative to the other sea-quarks. Instead, this measurement suggests the proton may be closer to an SU(3) flavor symmetric structure $(\bar{u}=\bar{d}=\bar{s})$ rather than a suppressed strange $\operatorname{PDF}(\bar{s}<\bar{u}, \bar{d})$.

The above case is just one example where improved determinations of the PDFs can yield superior precision for our theoretical predictions, and thus an enhanced ability to discern "new physics" signatures from uncertain "standard model" processes.

The goal of the nCTEQ collaboration is to make maximal use of the available data, both proton and nuclei, to obtain the most precise determination of the PDFs. In this brief report, we summarize some of the recent advancements toward this goal.

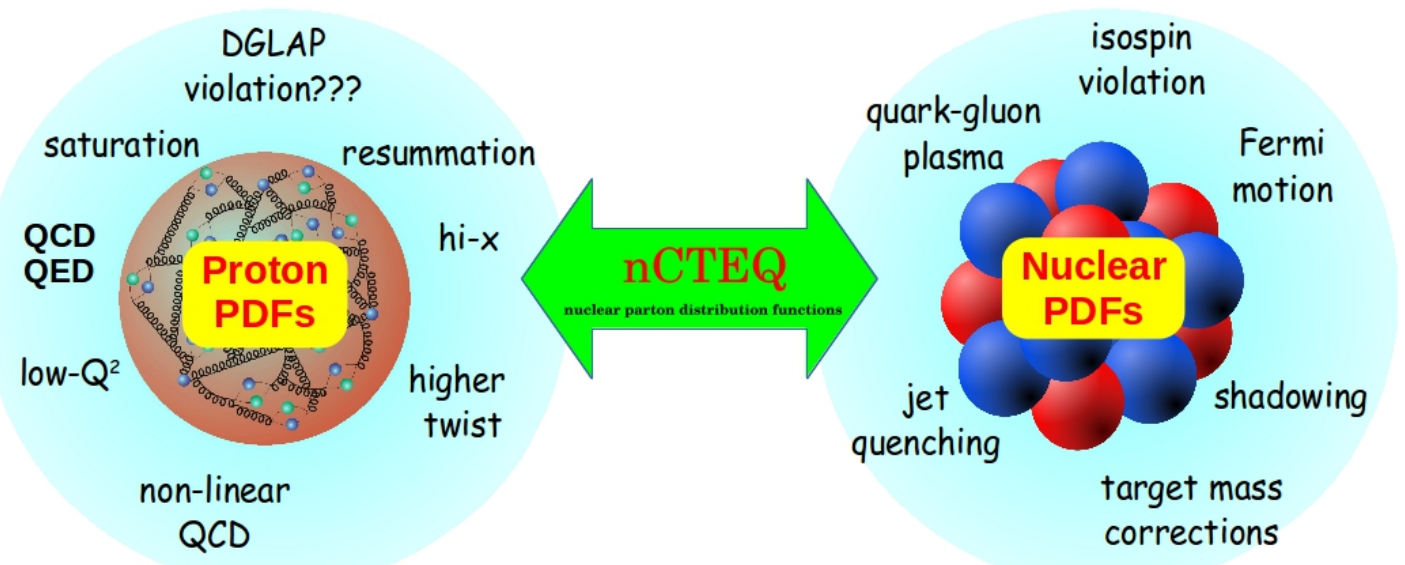

Figure 2: Schematic representation of selected phenomenological issues that can impact the determination of proton and nuclear PDFs.[1] 

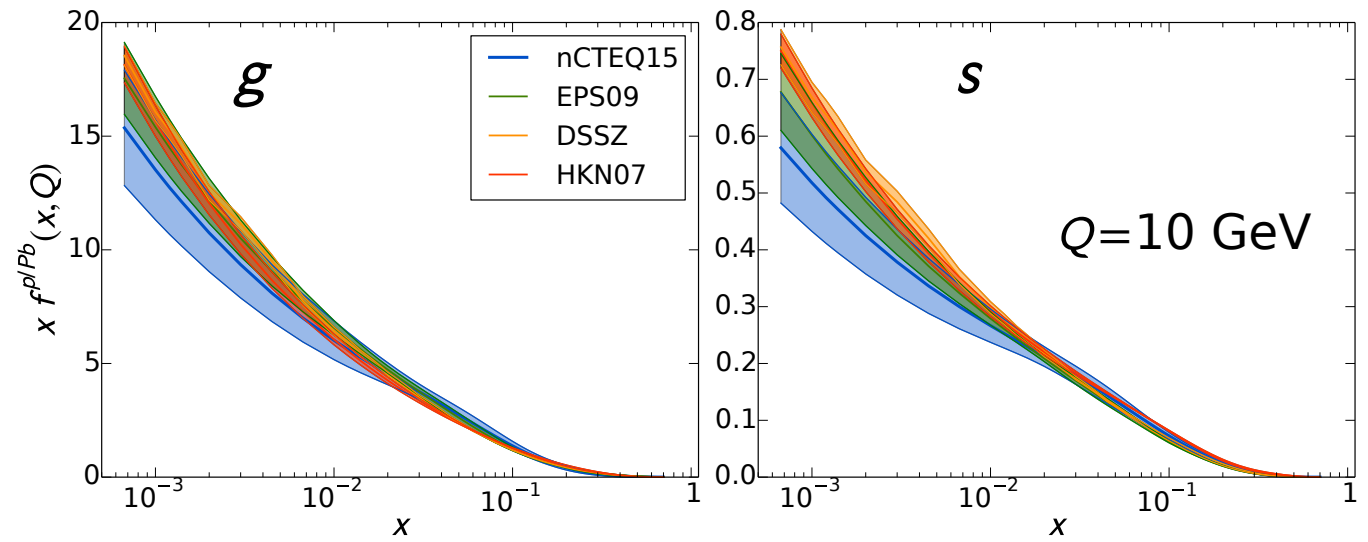

Figure 3: Selected PDF flavors from nCTEQ15 [1] compared with results from the literature [3-5].

\section{The nCTEQ Project}

The nCTEQ project ${ }^{1}$ built upon the work of the CTEQ proton PDF global fitting effort by extending the fit degrees of freedom into the nuclear dimension. Previous to the nCTEQ effort, nuclear data was "corrected" to isoscalar data and added to the proton PDF fit without any uncertainties. In contrast, the nCTEQ framework allows full communication between the nuclear data and the proton data, as illustrated schematically in Fig. 2. For example, this enables us to investigate if observed tensions between data sets could potentially be attributed to the nuclear corrections.

The details of the nCTEQ program is presented in Ref. [1]. Figure 3 displays selected fla-

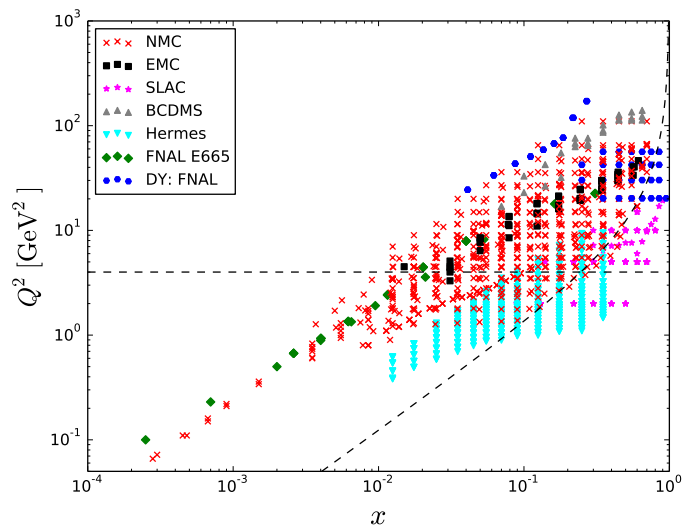

Figure 4: a) Kinematic reach of DIS and DY data used in the nCTEQ15 fits [1]. The dashed lines represent the kinematic cuts: $(Q>2 \mathrm{GeV}, W>3.5 \mathrm{GeV})$. vors from the nCTEQ15 set, and these compare favorably to other determinations from the literature [3-5]. This analysis used Deeply Inelastic Scattering (DIS), lepton pair production (Drell-Yan), and pion production from a variety of experiments totaling 740 data (after cuts) and 19 nuclei. The kinematic range of the data in the $\left\{x, Q^{2}\right\}$ plane is displayed in Fig. 4.

\section{LHC Heavy Ion $W$ Production}

Although the nCTEQ15 data covered an extensive range of kinematics and nuclear A, there is new heavy ion data from the LHC using proton-lead $(\mathrm{pPb})$ and lead-lead $(\mathrm{PbPb})$ collisions at even higher energies. We shall be interested to see how strongly this data can influence the nCTEQ15 PDFs. We will focus our discussion on the LHC heavy ion $W / Z$ rapidity distributions ${ }^{2}$ as we expect this can provide information on the (less constrained) strange and charm PDFs. The justification

\footnotetext{
${ }^{1}$ For details, see www . ncteq. org which is hosted at HepForge.org.

${ }^{2}$ For a more extensive study, see Refs. [7,8].
} 

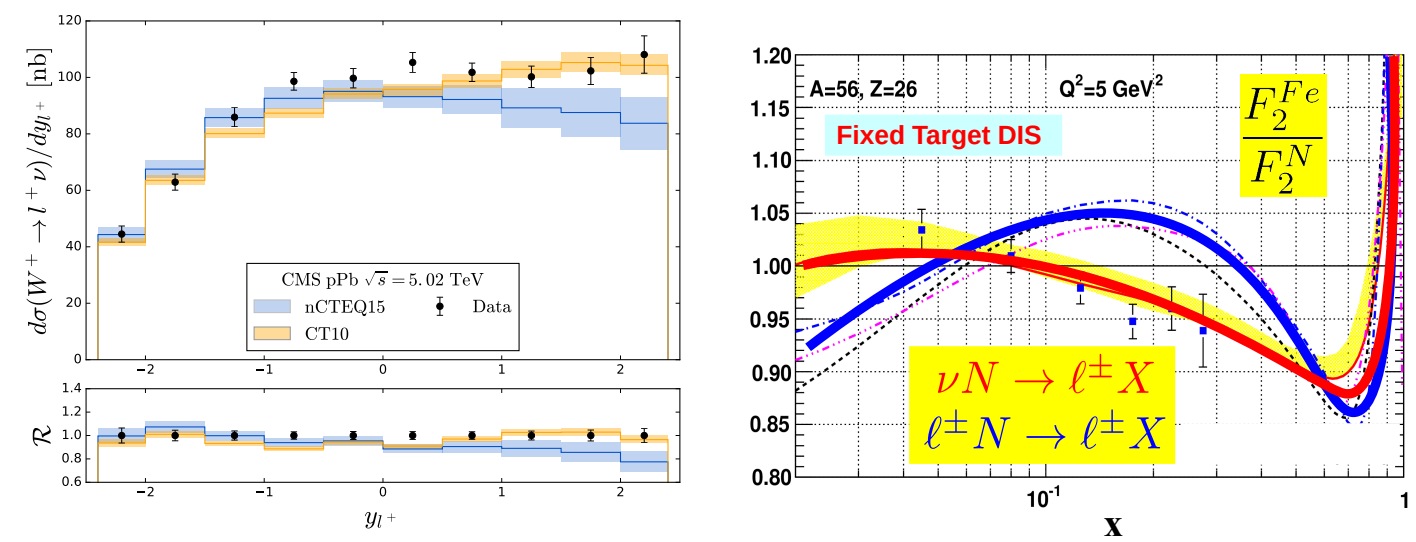

Figure 5: a) CMS $W^{+}$production in pPb collisions at the LHC [7].

b) The computed nuclear correction ratio, $F_{2}^{F e} / F_{2}^{D}$ as a function of $x$ for $Q^{2}=5 \mathrm{GeV}^{2}$, adapted from Ref. [6]. The blue line is for neutral current DIS charged-lepton scattering $\ell^{ \pm} N \rightarrow \ell^{ \pm} X$, and the red line is for charged current DIS neutrino scattering $v N \rightarrow \ell^{ \pm} X$.

for this expectation is presented in Ref. [9] which computes the relative partonic contributions for $W / Z$ production at the Tevatron $(p \bar{p})$ and the LHC $(p p)$. As the $\sqrt{s}$ increases, the heavier quarks $\{s, c, b\}$ provide an increased contribution to $W / Z$ production.

In Fig. 5-a) we display the CMS rapidity distribution for $W^{+}$production in $\mathrm{pPb}$ collisions with $\sqrt{s}=5.02 \mathrm{TeV}$. We show the results using the nuclear PDFs from nCTEQ15 (with nuclear corrections), and for comparison we also display CT10 PDFs (without nuclear corrections).

The kinematics of the process are defined such that negative rapidity corresponds to large lead $x$, and positive rapidity is small lead $x$. Thus, as we scan across the distribution in rapidity, we effectively scan in $x$. This is particularly evident when we compare nCTEQ15 with the CT10 results. At negative rapidity (large lead $x$ ) nCTEQ15 is above CT10, while at positive rapidity ( $\operatorname{small}$ lead $x$ ) nCTEQ15 is below. We compare this pattern with the expected nuclear correction as shown in Fig. 5-b). Focusing on the (blue) line for charged lepton DIS, we see the negative rapidity corresponds to the anti-shadowing region $(x \sim 0.2)$ where the nuclear PDFs are larger than the proton PDFs $\left(F_{2}^{F e}>F_{2}^{D}\right)$, while the positive rapidity corresponds to the shadowing region $(x \lesssim 0.05)$ where the nuclear PDFs are smaller than the proton PDFs $\left(F_{2}^{F e}<F_{2}^{D}\right)$. This very direct mapping between rapidity and $x$ can help us to better constrain the PDF $x$ distribution.

We now consider the comparison between the nCTEQ result and the displayed data. At negative rapidity (large lead $x$ ) the nCTEQ15 result compares well with the data, but at positive rapidity ( small lead $x$ ) the nCTEQ15 result significantly undershoots the data. Examining Fig. 4, we see that the bulk of the data used in nCTEQ15 (after cuts) is at relatively large $x$ values. Thus, the good comparison between nCTEQ15 and the data at large $x$ is encouraging. But the poor agreement at smaller $x$ suggests that the initial 740 data points used for the nCTEQ15 may not have been sufficient to constrain the small $x$ behavior; thus, including the new LHC heavy ion $W / Z$ data into the $\mathrm{nPDF}$ fit might help reduce the uncertainties in the small $x$ region.

There is an additional interesting observation we can make regarding Fig. 5-a); In Fig. 5-b), if we use the (red) neutrino DIS nuclear correction instead of the (blue) charged lepton result, the shadowing effect would be reduced and the nCTEQ15 result would move toward the data in the 
small $x$ region. The choice of which nuclear correction is correct has been discussed extensively in the literature, and there is no definitive resolution at present. ${ }^{3}$ Given that the LHC heavy ion $W / Z$ data is sensitive to the shadowing/anti-shadowing region, it could prove illuminating to include this into the fit.

\section{Cross Section Correlations}

The previous analysis suggests that new LHC heavy ion data can influence the nCTEQ15 PDFs. We would also like to specifically determine the impact of this data on the heavy flavors such as strange and charm. For this purpose, in Fig. 6 we display the correlation cross sections for $W^{ \pm}$ for 5 flavors of quarks $\{u, d, s, c, b\}$, and also for 2 flavors of quarks $\{u, d\}$. The obvious shift of these cases indicates the contribution of the heavy flavors $\{s, c, b\}$ is substantial; hence, these data are sensitive to, and can help constrain, the parton flavors. For both the 5 flavor and 2 flavor cases, we display 4 results; there are 3 results with nuclear corrections ${ }^{4}$ (nCTEQ15, CT10+EPS09, CTEQ6.1+ EPS09), and 1 result without nuclear corrections (CT10).

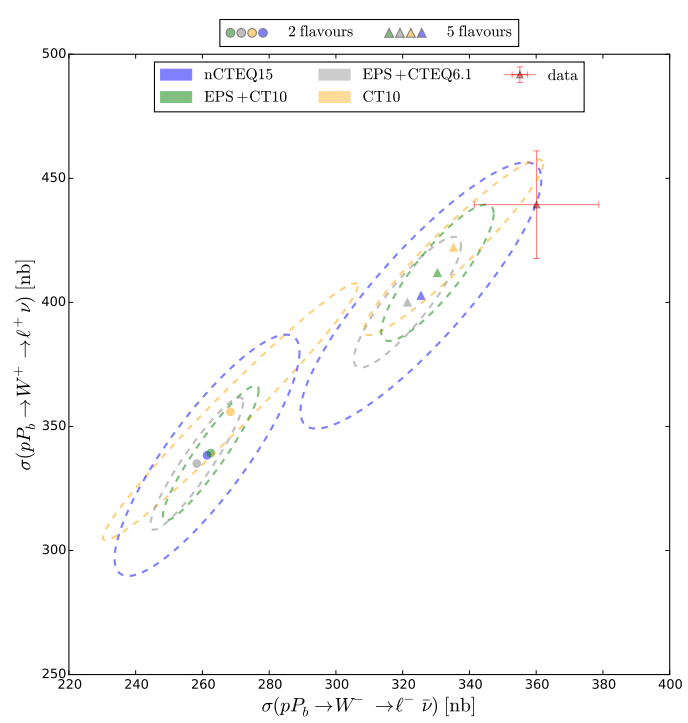

Figure 6: Comparison of correlations between $W^{+}$and $W^{-}$cross sections for the 5 flavor case $(\boldsymbol{\Lambda})$ and 2 flavor case $(\bullet)$. We show results for nCTEQ15, EPS09+CT10, EPS09+CTEQ6.1 and CT10 PDFs overlaid with the CMS data [7].

For the 5 flavor case, the calculations are scattered to the low side of the data in both $W^{+}$and $W^{-}$. The CT10 result is the closest to the data, but due to the larger uncertainties of nCTEQ15, the data point is within range of both of their ellipses. We also observe that the CT10+EPS09 and CTEQ6.1+EPS09 results bracket the nCTEQ15 value; this is due to the very different strange PDF associated with CT10 and CTEQ6.1.

For the 2 flavor case, all the nuclear results (nCTEQ15, CT10+EPS09, CTEQ6.1+EPS09) coalesce, and they are distinct from the non-nuclear result (CT10). This pattern suggests that the nuclear corrections of nCTEQ15 and EPS09 for the $\{u, d\}$ flavors are quite similar, and the spread observed in the 5 flavor case comes from differences of $s(x)$ in the underlying base PDF. ${ }^{5}$ Thus we infer that the difference between the nuclear results and the proton result accurately represents the nuclear corrections for the 2 flavor case (for $\{u, d\}$ ), but for the 5 flavor case it is a mix of nuclear corrections and variations of the underlying sea quarks.

\footnotetext{
${ }^{3}$ See Refs. [10,11], and also references therein.

${ }^{4}$ We have added CTEQ6.1+EPS09 as CTEQ6.1 was the baseline used for the EPS09 fit.

${ }^{5}$ While the charm PDF does play a role in the above (the bottom contribution is minimal), $c(x)$ is generated radiatively by the process $g \rightarrow c \bar{c}$ (we assume no intrinsic component) [12]; thus, it is essentially determined by the charm mass value and the gluon PDF. In contrast, the "intrinsic" nature of the strange PDF leads to its comparably large uncertainties. Therefore, the strange quark PDF will be primarily driving the observed differences.
} 


\section{Conclusion}

In this brief report we have observed that the LHC heavy ion data can help determine nuclear corrections for large A values in a kinematic $\left\{x, Q^{2}\right\}$ range very different from nuclear corrections provided by fixed-target measurements. The $W / Z \mathrm{pPb}$ data are sensitive to the heavier quark flavors (especially the strange PDF), so this provides important information on the nuclear flavor decomposition. Improved information on the nuclear corrections from the LHC lead data can also help reduce proton PDF uncertainties as (at present) fixed-target nuclear data is essential for distinguishing the individual flavors [13]. The next step is to incorporate this new data into the nPDF fit to help separately disentangle issues of flavor differentiation and nuclear corrections.

\section{References}

[1] K. Kovarik et al. [The nCTEQ Collaboration], nCTEQ15 - Global analysis of nuclear parton distributions with uncertainties in the CTEQ framework. Phys. Rev., D93(8):085037, 2016.

[2] Morad Aaboud et al. Precision measurement and interpretation of inclusive $W^{+}, W^{-}$and $Z / \gamma^{*}$ production cross sections with the ATLAS detector. Eur. Phys. J., C77(6):367, 2017.

[3] M. Hirai, S. Kumano, and T. H. Nagai. Determination of nuclear parton distribution functions and their uncertainties in next-to-leading order. Phys. Rev., C76:065207, 2007.

[4] K. J. Eskola, H. Paukkunen, and C. A. Salgado. EPS09: A New Generation of NLO and LO Nuclear Parton Distribution Functions. JHEP, 04:065, 2009.

[5] Daniel de Florian, Rodolfo Sassot, Pia Zurita, and Marco Stratmann. Global Analysis of Nuclear Parton Distributions. Phys. Rev., D85:074028, 2012.

[6] I. Schienbein, J. Y. Yu, K. Kovarik, C. Keppel, J. G. Morfin, F. Olness, and J. F. Owens. PDF Nuclear Corrections for Charged and Neutral Current Processes. Phys. Rev., D80:094004, 2009.

[7] A. Kusina, et al. [The nCTEQ Collaboration], Vector boson production in proton-lead and lead-lead collisions at the LHC and its impact on nCTEQ15 PDFs. Eur. Phys. J., C77(7):488, 2017.

[8] Kari J. Eskola, Petja Paakkinen, Hannu Paukkunen, and Carlos A. Salgado. EPPS16: Nuclear parton distributions with LHC data. Eur. Phys. J., C77(3):163, 2017.

[9] A. Kusina, T. Stavreva, S. Berge, F. I. Olness, I. Schienbein, K. Kovarik, T. Jezo, J. Y. Yu, and K. Park. Strange Quark PDFs and Implications for Drell-Yan Boson Production at the LHC. Phys. Rev., D85:094028, 2012.

[10] K. Kovarik, I. Schienbein, F. I. Olness, J. Y. Yu, C. Keppel, J. G. Morfin, J. F. Owens, and T. Stavreva. Nuclear corrections in neutrino-nucleus DIS and their compatibility with global NPDF analyses. Phys. Rev. Lett., 106:122301, 2011.

[11] Hannu Paukkunen and Carlos A. Salgado. Agreement of Neutrino Deep Inelastic Scattering Data with Global Fits of Parton Distributions. Phys. Rev. Lett., 110(21):212301, 2013.

[12] Florian Lyonnet, Aleksander Kusina, Tomáš Ježo, Karol Kovarík, Fred Olness, Ingo Schienbein, and Ji-Young Yu. On the intrinsic bottom content of the nucleon and its impact on heavy new physics at the LHC. JHEP, 07:141, 2015.

[13] R. D. Ball et al. [NNPDF Collaboration], Parton distributions from high-precision collider data, Eur. Phys. J., C77(10):663, 2017. 